\title{
Research On SMS Filtering Algorithm Based On User Personality
}

\author{
Wenli Gong,Guojiang Fu \\ School of computer science and technology, Wuhan University of Technology, Wuhan,430070,China
}

Keywords: User feature modeling; Personalise; SMS filtering

\begin{abstract}
In this paper, a short message filtering model based on the user characteristic model is proposed to solve the problem that the short message filter model is not constructed from the user's needs. The model analyzes the information of App category and user's existing SMS in the user's mobile phone, and carries on the user characteristic modeling. And uses the obtained user feature model to optimize the SMS filtering algorithm, and then obtains the user-based SMS filtering model, which can achieve the accuracy of SMS filtering, but also meet the user's personalized SMS filtering needs. Finally, the rationality and validity of the model are proved by experiments.
\end{abstract}

\section{Introduction}

The existing improvements to the SMS filtering algorithm are aimed at the traditional sense of spam messages. In the process of improvement, it does not take into account the user's definition of spam messages, and does not construct the SMS filter from the user's needs and the SMS filter lacks of personalise. Aiming at this problem, this paper proposes a SMS filtering model based on the user characteristic model. The model analyzes the information of App category and user's existing SMS in the user's mobile phone, and obtains the user characteristic model. And uses the model to optimize the SMS filtering algorithm, and obtains the SMS filtering model based on the user features.

\section{User Feature Model Based on App Classification}

Users operations of install,sharing or uninstall with their smart phone Apps reflects the user's interest to a certain extent. So this section makes use of App's category attribute information, the number of times the user accesses the App, whether the user has shared the App and whether the user has uninstalled the App to construct the user's user -App score array $\mathrm{R}$ and the App-class matrix $\mathrm{Q}$, and use them to construct the user's interest model $\mathrm{M}$.

The user -App score array $\mathrm{R}=\left[\mathrm{r}_{\mathrm{Al}}, \mathrm{r}_{\mathrm{A} 2}, \ldots, \mathrm{r}_{\mathrm{An}}\right]$ where $\mathrm{r}_{\mathrm{Ai}}$ represents the real score of the application Ai.In this paper, the user of the statistical data in the App rating is set to $[0,1,2,3,4,5]$ six levels, the higher the score that the users more interested in the Apps ${ }^{[1]}$. Specifically, a value of 0 indicates that the user has uninstalled the application. Scoring based on the mainly include: installation, use, frequency of sharing and uninstall.

The Apps which the user has access to get their points by the following rules :

a) When an App is installed but did not use by the user,give the app point 1 ;

b) When Apps are used by user,give them the point between 2 to 5 according to the use of times;

c) When user share an App,give it score of 5, indicating that the user is very interested in the App;

d) When user uninstall an App,give it 0 points indicates that the user is not interested in the app at all.

Each application has its own category attribute information. In this paper, we denote the category attribute of App by using $\mathrm{C}\left\{\mathrm{c}_{1}, \mathrm{c}_{2}, \mathrm{c}_{3}, \ldots, \mathrm{c}_{\mathrm{k}}\right\}$, the attribute attribute matrix $\mathrm{Q}$ Expressed as:

$Q=\left|\begin{array}{l}q_{A_{1}, C_{1}}, q_{A_{1}, C_{2}}, q_{A_{1}, C_{3}}, q_{A_{1}, C_{4}}, q_{A_{1}, C_{5}}, \ldots, q_{A_{1}, C_{k}} \\ q_{A_{2}, C_{1}}, q_{A_{2}, C_{2}}, q_{A_{2}, C_{3}}, q_{A_{2}, C_{4}}, q_{A_{2}, C_{5}}, \ldots, q_{A_{2}, C_{k}} \\ q_{A_{3}, C_{1}}, q_{A_{3}, C_{2}}, q_{A_{3}, C_{3}}, q_{A_{3}, C_{4}}, q_{A_{3}, C_{5}}, \ldots, q_{A_{3}, C_{k}} \\ \ldots \ldots . . \ldots \ldots . . \ldots \ldots . . \ldots \ldots \ldots, \ldots \ldots \ldots \ldots, \ldots \ldots . \\ q_{A_{n}, C_{1}}, q_{A_{n}, C_{2}}, q_{A_{n}, C_{3}}, q_{A_{n}, C_{4}}, q_{A_{n}, C_{5}}, \ldots, q_{A_{n}, C_{k}}\end{array}\right|$

where $\mathrm{q}_{\mathrm{Ai}, \mathrm{Ci}}$ represents whether the application $\mathrm{A}_{\mathrm{i}}$ belongs to the app category $C_{j}$.If $A_{i}$ belongs to $C_{j}, q_{A i, C i}=1$, else $q_{A i, C i}=0$.

Then calculates the user's preference matrix $M$ using Equation 1: 


$$
\mathrm{M}=\mathrm{RQ}
$$

\section{User Feature Modeling Based on Short Message Class}

Existing mobile phone text messages in the user's phone contains a wealth of content, such as SMS text content, send text messages, SMS receiving time, and SMS reading time and other information. If take full advantage of these messages, we can analysis the interest of user more accurately, and then get a more accurate user interest model. Based on this, this section extracts the topic information from the user SMS content based on the NaiveBayes ${ }^{[2]}$ algorithm, and calculates the messages' category for the existing short messages,then calcutes the interest model.

Naive Bayes algorithm is based on the Bayesian theorem and feature condition independent hypothesis classification $\operatorname{method}^{[3]}$,in the classification process,calculating the probability of each sms category,and this text is classified in the category whose probability is the largest ${ }^{[4]}$.

The classification process of Naive Bayes algorithm is as follows:

(1) Suppose that the sms $d=\left\{t_{1}, t_{2}, t_{3}, \ldots, t_{n}\right\}$ is an item to be categorized, where $t_{i}$ represents the characteristic attribute of the message $\mathrm{d}$;

(2) There is a sms category set $\mathrm{C}=\left\{\mathrm{c}_{1}, \mathrm{c}_{2}, \mathrm{c}_{3}, \ldots, \mathrm{c}_{\mathrm{k}}\right\}$;

(3) Calculate $P\left(c_{j} \mid d\right)$ for each class $c_{j}$;

(4) If the category $c_{m}$ has the maximum posteriori probability, the category of the sms $d$ is $c_{m}$.

In the process of classifying the existing sms in the user's mobile phone, the messages are divided into two test sets according to the read status and the unread status. After pre-processing operations such as word segmentation, deactivation words, feature representation and feature extraction, the short text of each test set is calculated based on the Naive Bayes algorithm, and counted the probabilities $\mathrm{P}\left(\mathrm{c}_{\mathrm{j}}\right)$ using Equation 2, where A represents the count of items in category $c_{j}$ and $B$ represents the total count of the test dataset:

$$
P\left(c_{j}\right)=\frac{A}{B}
$$

After counting, we can calculate the user's interest model: $\mathrm{D}=\alpha \mathrm{D}_{\mathrm{R}}+\beta \mathrm{D}_{\mathrm{U}} \cdot$ where

$\mathrm{D}_{\mathrm{R}}=\left[\mathrm{P}\left(\mathrm{c}_{1}\right), \mathrm{P}\left(\mathrm{c}_{2}\right), \ldots, \mathrm{P}\left(\mathrm{c}_{\mathrm{i}}\right)\right], \mathrm{D}_{\mathrm{U}}=\left[\mathrm{P}\left(\mathrm{c}_{1}\right), \mathrm{P}\left(\mathrm{c}_{2}\right), \ldots, \mathrm{P}\left(\mathrm{c}_{\mathrm{j}}\right)\right]$

\section{SMS Filtering Model Based on User Feature}

\section{Model}

SMS filtering model combines the black and white list mechanism and naive Bayesian classification algorithm.Suppose $d=\left\{d_{\text {time }}, d_{\text {tel }}, d_{\text {content }}\right\}$, where $d_{\text {time }}$ is the receiving time of the $s m s d, d_{\text {tel }}$ is the sending number of the sms $d, d_{\text {content }}$ is the text content of the sms $d$. Then the process to determine whether the user wants to see the message is as follows:

(1) Determine whether $d_{\text {tel }}$ in the user's white list. If it exists, the message will be stored directly into the SMS inbox, and end the filtering process, otherwise proceed to step 2;

(2) Determine whether $d_{\text {tel }}$ in the user's blacklist. If it exists, it will be directly stored in the SMS trash can, and end the filtration process, otherwise proceed to step 3;

(3) Get the message text's feature set $\{t 1, t 2, \ldots, t n\}$;

(4) Determine whether the feature set of the text message contains any application name in the user's mobile phone If included, directly compares to the user characteristics of the model $\mathrm{M}$ based on the App category to find the corresponding interest in the App. If the degree of interest is greater than or equal to a certain threshold, the message is judged to be the message that the user wants to see, and stored in the inbox of the user's mobile phone; otherwise, the message is judged as the message that the user does not want to see. If not, proceed to step 5.

(5) Calculate the category of sms d ;

(6) Compare to the user characteristic model D based on the short message category to find the user's interest degree. If the interest degree is greater than or equal to a certain threshold, the short message is judged as the user wants to see the message, and stored in the user's mobile phone, Otherwise, the message is judged as the user does not want to see the message, save it in the user's mobile phone's trash can.

\section{Experiment results and analysis}

\subsection{Experiment Dataset}

Currently there is no public text corpus dedicated to SMS classification $^{[5]}$, so the experiment is carry on a 
self-collection of SMS training with 11,000 messages.The dataset for establishing the user characteristics model is the App information and existing SMS got form the user's phone. The SMS test set for the SMS filtering phase is the 5500 messages provides by volunteers. The number of messages marked "Users want to see" is 4000 , and the number of messages marked "Users do not want to see" is 1500.

\subsection{Experiment evaluation criteria}

At this stage of the spam message filter performance evaluation generally use the following three indicators ${ }^{[3]}$ :

(1) Recall: marked the classfier's filter capacity, the greater the value, the "false negatives" less.

(2) Precision: mark the ability to identify spam messages, the greater the value, the less likely to misjudge.

(3) F-Score:F-score is the harmonized average of accuracy rate and recall rate.

Set Table I before the calculation so that it can be referenced in the formula:

\begin{tabular}{|c|c|c|}
\hline & Legal SMS & Spam SMS \\
\hline $\begin{array}{c}\text { Determined to } \\
\text { be legal SMS }\end{array}$ & $\mathrm{N}_{\mathrm{T}->\mathrm{T}}$ & $\mathrm{N}_{\mathrm{F}->\mathrm{T}}$ \\
\hline $\begin{array}{l}\text { Determined to } \\
\text { be spam SMS }\end{array}$ & $\mathrm{N}_{\mathrm{T}-\mathrm{F}}$ & $\mathrm{N}_{\mathrm{F}->\mathrm{F}}$ \\
\hline
\end{tabular}

Table I: Variable definition table

$$
\begin{aligned}
& P=\frac{N_{T->T}}{N_{T->T}+N_{F->T}} * 100 \% \\
& R=\frac{N_{T->T}}{N_{T->T}+N_{T->F}} * 100 \% \\
& F-\text { score }=\frac{2 * P * \mathrm{R}}{\mathrm{P}+\mathrm{R}}
\end{aligned}
$$

\subsection{Analysis of results}

In order to compare the results of mobile spam filtering algorithm based on user's personality characteristics, the short message filtering model based on Naive Bayes algorithm is adopted as the contrast model. The results of their respective detection are shown in Table II.

\begin{tabular}{|c|c|c|c|c|c|}
\hline $\begin{array}{c}\text { Meth- } \\
\text { od }\end{array}$ & $\mathrm{N}_{\mathrm{T}>\mathrm{T}}$ & $\mathrm{N}_{\mathrm{F}-\mathrm{T}}$ & $\mathrm{P}(\%)$ & $\mathrm{R}(\%)$ & $\mathrm{F}(\%)$ \\
\hline $\begin{array}{c}\text { Intere } \\
\text { st } \\
\text { Mode } \\
1\end{array}$ & 3746 & 153 & 96.08 & 93.65 & 94.85 \\
\hline $\mathrm{NBC}$ & 3168 & 442 & 87.77 & 79.20 & 83.27 \\
\hline
\end{tabular}

Table II:Comparison of Result of SMS Filtering Algorithm

From the comparison results in Table 2, we can see that the filtering accuracy and recall rate of the SMS model based on the user's personality are much higher than those based on the single naive Bayes algorithm. The main reasons are as follows: The short message filtering model which features black and white list technology as the first line of defense against spam detection can identify the spam messages sent by the known spam message phone number. Second, the short message filtering model based on the user's personalities features the user interest model combined the short message filtering with user interest model,and the results of filtering are more close to the user's real needs, which effectively improve the spam filtering accuracy.

\section{Conclusion}

In this paper, a short message filtering model based on user characteristic model is proposed to solve the problem that the existing spam filtering model does not construct the SMS filtering model from the user's needs. The model's advantage compares to the traditional short message filtering model is that it establishes the user characteristic model according to the acquired information and the existing short message information of the user's mobile phone, and judges whether the new incoming message is the messages which user wants to see.The model meets the user's personalized SMS filtering needs. The results show that the accuracy and recall rate of the model are significantly higher than the traditional SMS filtering model. 


\section{References}

[1] Cheng Zhou. Research on recommendation system based on cloud computing [D]. Wuhan: Wuhan University of Technology, 2014.

[2] Guangwei Ge.Application of the classification of inspam short message filtering [D]. Yunnan University of Finance and Economics, 2015.

[3] Li Hang. Statistical learning method [M]. Tsinghua University press, 2012.

[4] Cheng Huang.Research on the Filtering Technology of Smart Phone's Junk Message[D].Huazhong University of Science and Technology,2012.

[5] Qiaokou Zhou.research and exploration of a kind of spam filtering algorithm based on semantics[J].laboratory,2016,35 (11): 137-140. 